\title{
Treatment of the Initial Value in Time-Memory-Data Tradeoff Attacks on Stream Ciphers
}

\author{
Orr Dunkelman ${ }^{1, \star}$ and Nathan Keller ${ }^{2, \star \star}$ \\ ${ }^{1}$ Katholieke Universiteit Leuven \\ Department of Electrical Engineering ESAT/SCD-COSIC \\ Kasteelpark Arenberg 10, B-3001 Leuven-Heverlee, Belgium \\ orr.dunkelman@esat.kuleuven. be \\ ${ }^{2}$ Einstein Institute of Mathematics, Hebrew University. \\ Jerusalem 91904, Israel \\ nkeller@math.huji.ac.il
}

\begin{abstract}
Time-Memory Tradeoff (TMTO) attacks on stream ciphers are a serious security threat and the resistance to this class of attacks is an important criterion in the design of a modern stream cipher. TMTO attacks are especially effective against stream ciphers where a variant of the TMTO attack can make use of multiple data to reduce the off-line and the on-line time complexities of the attack (given a fixed amount of memory).

In this paper we present a new approach to TMTO attacks against stream ciphers using a publicly known initial value (IV): We suggest not to treat the IV as part of the secret key material (as done in current attacks), but rather to choose in advance some IVs and apply a TMTO attack to streams produced using these IVs. We show that while the obtained tradeoff curve is identical to the curve obtained by the current approach, the new technique allows to mount the TMTO attack in a larger variety of settings. For example, if both the secret key and the IV are of length $n$, it is possible to mount an attack with data, time, and memory complexities of $2^{4 n / 5}$, while in the current approach, either the time complexity or the memory complexity is not less than $2^{n}$.
\end{abstract}

Keywords: Time-Memory-Data Tradeoff attacks, Stream ciphers, IV initialization.

\section{Introduction}

The problem of inverting a one-way function is a basic problem in cryptanalysis. For example, the problem of deducing the encryption key from a plaintext/ciphertext pair can be modeled as such. Two extreme solutions of the

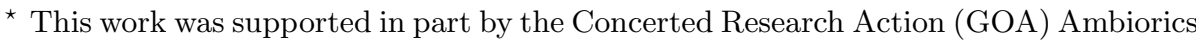
2005/11 of the Flemish Government and by the IAP Programme P6/26 BCRYPT of the Belgian State (Belgian Science Policy).

** This author is supported by the Adams Fellowship Program of the Israel Academy of Sciences and Humanities.
} 
problem are exhaustive key search and the table attack (also called the dictionary attack). In exhaustive key search, the time complexity of the attack is $N$ encryptions (where $N$ is the number of possible keys), the memory requirement is negligible, and there is no preprocessing phase. In the table attack, the time complexity of the on-line phase of the attack is negligible, while the memory requirement is $N$ memory cells, and a one-time preprocessing phase with time complexity of $N$ encryptions is required.

In [12] Hellman presented a trade-off between the two attacks described above. Denoting the online time complexity of the attack by $T$ and the memory requirements by $M$, the attack satisfies the relation $N^{2}=T M^{2}$. Thus, a convenient choice of $M$ and $T$ is $M=T=N^{2 / 3}$. The time complexity of the pre-processing phase is $N$ encryptions. Later, Biryukov and Shamir [6] showed that if the attacker has access to multiple data points, the tradeoff curve obtained in Hellman's attack can be improved. If the number of available data points is $D$, the attacker can produce an attack which satisfies $N^{2}=T M^{2} D^{2}$, and has a preprocessing step of $N / D$ operations. These curves can be achieved under various conditions, e.g., the attack from [6] requires $T \geq D^{2}$.

Hellman's ideas, originally proposed for attacking DES, were extended to various cryptosystems. Probably the most successful extension is the application of TMTO to stream ciphers. Several stream ciphers were broken using TMTO [5, 16], and resistance to TMTO attacks is considered an important criterion in stream cipher design [9].

TMTO attacks on stream ciphers can be divided into two classes, according to the one-way function the attacker tries to invert. The first class of attacks (denoted in the sequel Scenario $A$ attacks) tries to invert the function (Internal State $\rightarrow$ Output Prefix) $[1,6,11]$. The second class of attacks (denoted in the sequel Scenario $B$ attacks) tries to invert the function (Secret Key $\rightarrow$ Output Prefix) $[13,14]$.

In this paper we consider Scenario B attacks on stream ciphers with IV. We propose a new approach to attacks of this class: Instead of inverting the function ((Key,IV) $\rightarrow$ Output Prefix), we suggest to choose in advance several IVs, compute the TMTO tables for the function (Key $\rightarrow$ Output Prefix) for each of the chosen IVs, and apply Hellman's original attack on encryptions using the chosen IVs.

We show that the tradeoff curve obtained for the new approach is $N^{2}=$ $T M^{2} D^{2}$, where $N$ is the number of possible keys multiplied by the number of possible IVs, and $D$ is the amount of data available to the attacker. Hence, the tradeoff curve is the same as the tradeoff curve obtained by the current approach.

However, we show that the new approach has several advantages. The main advantage is that while the current approach allows to obtain the tradeoff curve $N^{2}=T M^{2} D^{2}$ only for $T \geq D^{2}$, the new approach allows to obtain this curve for all values of $D$. For example, if the key size is 64 bits, the IV size is 40 bits, and the amount of available data is $D=2^{32}$, it is possible to mount a TMTO attack with $T=M=2^{48}$, while in the current approach, the parameters of the attack must satisfy the inequality $T M \geq 2^{104}$. 
Moreover, we show that if the length of the secret key is $n$ bits and the IV length is less than $1.5 n$ bits, the new approach allows to mount an attack with data, memory, and time complexities less than $2^{n}$. In particular, setting the IV length to be equal to the key length does not ensure an $n$-bit security against TMTO attacks, contrary to $[4,8,13,14]$. For example, if both the secret key and the IV are of length $n$, the new approach allows to mount an attack with data, time, and memory complexities of $2^{4 n / 5}$.

Finally, we discuss cases where the IV update scheme is (even partially) deterministic. We show that this common approach is less secure than picking the IV at random each and every time.

The rest of the paper is organized as follows: In Section 2 we briefly describe the basic ideas of time-memory-data tradeoffs. In Section 3 we present our new approach and its advantages. We summarize the paper in Section 4.

\section{A Brief Overview of Time-Memory-Data Tradeoffs}

In this section we briefly overview some of the previously known TMTO attacks. A more extensive summary of TMTO attacks can be found in [3].

\subsection{Hellman's and Oechslin's TMTO Attacks}

We start with the basic TMTO attack of Hellman [12]. Let $f:\{0,1, \ldots, N-1\} \rightarrow$ $\{0,1, \ldots, N-1\}$ be the function the attacker tries to invert.

The pre-processing phase of the attack consists of constructing several tables. To construct each table, the attacker chooses $m$ random starting points, and from each starting point $x$ she computes the chain $S P=x, f(x), f^{2}(x)=$ $f(f(x)), \ldots, f^{t}(x)=E P$, as shown in Figure 1, (where $f$ is the function the attacker tries to invert). The pairs $(E P, S P)$ are stored in a table. The attacker constructs $t$ such tables $T_{0}, \ldots T_{t-1}$, each for a different function $f_{i}$ that is usually a slight modification of the original $f$ (e.g., a permutation of the bits). In the on-line phase of the attack, the attacker is given $z=f(y)$ and has to find $y$. For all $0 \leq i \leq t-1$, she applies $f_{i}$ repeatedly to $f_{i}(y)$ (that can be easily computed given $f(y))$ to get the sequence $f_{i}(y), f_{i}^{2}(y), \ldots, f_{i}^{t}(y)$, for each new value the attacker checks whether the obtained value is an end point in the table $T_{i}$. If a value appears as an end point in the table, the attacker takes the corresponding starting point and applies $f_{i}$ sequentially, until $f_{i}(y)$ is reached. The point encountered just before $f_{i}(y)$ is indeed $y$.

The time complexity of the attack is $t^{2}$ applications of $f$ and $t^{2}$ database accesses, and the memory required for the attack is $m t$. Since the tables should cover most of the $N$ possible states, we have $N=m t^{2}$, and hence $N^{2}=T M^{2}$ is the tradeoff curve obtained for this attack. The time complexity of the preprocessing phase is $N$, but as we noted before, this phase is usually neglected in the analysis of TMTO attacks.

In 2003, Oechslin [15] presented a different method to construct the tables in the TMTO attack. In the new method, a single table (called the Rainbow table) is 


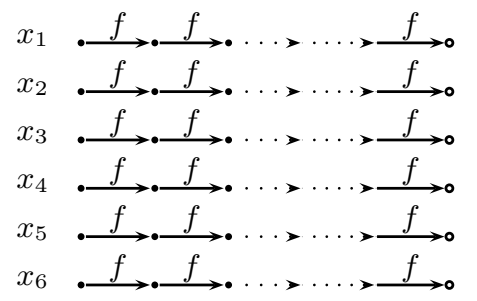

Fig. 1. Constructing Hellman's Table

constructed. This time, $m t$ starting points are chosen, and the constructed chains are of the form $x, f_{0}(x), f_{1}\left(f_{0}(x)\right), f_{2}\left(f_{1}\left(f_{0}(x)\right)\right), \ldots, f_{t-1}\left(\ldots\left(f_{0}(x)\right)\right)$, where the functions $f_{i}$ are the same as used in Hellman's scheme. This technique reduces the effect of collisions in the table, and hence allows to cover most of the state space by a single table.

On the other hand, the on-line phase of the attack is more complicated. First, the attacker checks whether $f_{t-1}(y)$ (computed from the given $f(y)$ ) appears as an end point in the table. If not, she computes $f_{t-1}\left(f_{t-2}(y)\right)$ and checks whether this value appears in the table. If not, she computes $f_{t-1}\left(f_{t-2}\left(f_{t-3}(y)\right)\right)$, and so on. Once an end point is encountered, the attack proceeds as Hellman's attack.

The time complexity of the attack is $1+2+\ldots+(t-1) \approx t^{2} / 2$ applications of $f$ and $t$ database accesses, and the memory requirement is $m t$. Since this time most of the states are covered by the single table, we have $N=m t^{2}$, and hence the obtained tradeoff curve is $N^{2}=2 T M^{2}$.

\subsection{Time-Memory-Data Tradeoff Attacks}

In 2000, Biryukov and Shamir showed that if the attacker has access to multiple data points, the tradeoff curve obtained in Hellman's attack can be improved [6]. ${ }^{1}$ It is important to note that the multiple data points stand for pairs of (unknown input, known output) to the function that the attacker tries to invert.

If the number of available data points is $D$, the attacker can construct tables that cover only $N / D$ of the $N$ states, and by the birthday paradox one of the data points falls into the covered states. In the on-line phase of the attack, the attacker repeats the attack for all the data points, and once one of the points is covered by the table, the secret key can be retrieved (assuming that the attacker is satisified with inverting only one of the obtained values).

In this case there are only $t / D$ tables, and thus the time complexity of the attack remains $t^{2}$ while the memory requirement is reduced to $m t / D$. Since this time we have $N / D=m t^{2}$, the obtained tradeoff curve is $N^{2}=T M^{2} D^{2}$. The time complexity of the pre-processing phase is reduced to $N / D$.

\footnotetext{
${ }^{1}$ We note that the idea of using multiple data points in TMTO attacks on stream ciphers was introduced in [1] and [11]. Biryukov and Shamir [6] were the first to combine this idea with Hellman's attack.
} 
It is noted in [6] that the tradeoff curve $N^{2}=T M^{2} D^{2}$ can be obtained only if $T \geq D^{2}$, since only in this case at least one "full" table consisting of $m$ chains of length $t$ each, where $m t^{2}=N$, can be constructed. If $T<D^{2}$ and a single "smaller" table is used in the attack, the resulting time complexity is $D t$ and the memory requirement is $m$. However, since in this case we have $N / D=m t$, the obtained tradeoff curve is $T M^{2} D^{2}=D^{3} t m^{2}>D^{2} t^{2} m^{2}=D^{2}(N / D)^{2}=N^{2}$ (where we used the inequality $D>t$ following from the assumption $D^{2}>T$ ).

In [4] the technique presented in [6] is extended to the case $T<D^{2}$ and various tradeoff curves are suggested. However, all the suggested curves are inferior to the curve $N^{2}=T M^{2} D^{2}$.

The usage of multiple data points in the Rainbow scheme is examined in [2, 4]. In [4] it is shown that a direct application of the Rainbow scheme in the case of multiple data points leads to the inferior tradeoff curve $N^{2}=T M^{2} D$. In [2] two more complicated algorithms are presented. The first leads to the tradeoff curve $N^{2}=T M^{2} D^{2}$, thus losing the factor two advantage of the Rainbow scheme. The second yields the curve $N^{2}+N D^{2} M=2 T M^{2} D^{2}$. This allows to save the factor two advantage if $T \gg D^{2}$, but if $T$ is close to $D^{2}$, most of the gain of the Rainbow scheme is lost.

\subsection{TMTO Attacks on Stream Ciphers}

TMDTO attacks on stream ciphers can be divided into two classes, according to the one-way function the attacker tries to invert: Inverting the internal state to output transformation, and inverting the (key, IV) to output transformation.

The first class of attacks (i.e., Scenario A attacks) is presented in $[1,5,6,11]$. These attacks try to invert the function (Internal State $\rightarrow$ Output Prefix). More precisely, to retrieve an internal state of $s$ bits, the attacker use a mapping of these $s$ bits to $s$ bits of the output stream.

In $[1,11]$ the attacker samples the output stream produced by (randomly chosen) internal states. Then, each real output stream is searched for in the generated list. If a match is found, the internal state is (with very high probability) the one that was sampled.

It is also possible to use Hellman's ideas in Scenario A attacks. In these attacks the trade-off curve provided by Hellman's basic attack can be improved using multiple data points. If the number of available data points is $D$, the attacker can construct tables that cover only $N / D$ of the $N$ states, and by the birthday paradox one of the data points falls into the covered states [6]. In the on-line phase of the attack, the attacker repeats the attack for all the data points, and once one of the points is covered by the table, the secret key can be retrieved. Each additional output bit provides the attacker with additional data point, and if the attacker uses $D$ such points, the tradeoff curve can be improved to $N^{2}=T M^{2} D^{2}$, for $T \geq D^{2}$. Attacks of this class were used to break several stream ciphers, including A5/1 and LILI-128 [5, 16]. A countermeasure against Scenario A attacks, deployed in most modern stream ciphers, is using a large internal state that makes this class of attacks less favorable than exhaustive key search. 
The second class of attacks (i.e., Scenario B attacks) is presented in [13]. These attacks try to invert the function (Secret Key $\rightarrow$ Output Prefix).

Scenario B attacks cannot use multiple data points from the same output stream, but the on-line time complexity of the attacks is less than that of exhaustive key search, independently of the size of the internal state. The security of a stream cipher against Scenario B attacks is increased if the cipher uses a publicly known Initial Value (IV). If the attacker does not know the IV in advance, she cannot use it in the pre-processing phase of the TMTO attack. The current approach in this situation is to treat the IV as part of the secret key [4, $13,14]$. As a result, the amount of secret key material is increased, but at the same time, the attack can use multiple data points. The data points are obtained from encryptions using the same secret key and different IVs. ${ }^{2}$ As a result, the tradeoff curve $N^{2}=T M^{2} D^{2}$, for $T \geq D^{2}$, is again possible, where $N$ is the number of possible keys multiplied by the number of possible IVs. In order to prevent Scenario B attacks, as well as other attacks, it is suggested in $[4,8,13$, $14]$ to require stream ciphers to have IV at least as long as the secret key.

\section{A New Approach to TMTO Attacks on Stream Ciphers Using Initial Values}

In this section we present our new approach to Scenario B TMTO attacks on stream ciphers with IV.

We observe that the current approach, i.e., treating the IV as part of the secret key and trying to invert the function ((Key,IV) $\rightarrow$ Output Prefix) is not optimal, since it does not exploit the fact that the IV is publicly known. We propose to choose in advance several IVs and try to invert the function (Key $\rightarrow$ Output Prefix) for the chosen IVs. ${ }^{3}$

Denote the number of possible secret keys by $K$ and the number of possible IVs by $V$. Assume that the amount of data (i.e., output streams generated under the secret key with different IVs) available in the on-line phase of the attack is $D \leq V$.

In the pre-processing phase of the attack, the attacker chooses $V / D$ IVs. For each chosen $I V_{i}$, the attacker prepares Hellman's tables (or Rainbow tables) to invert the function $f:$ (Key $\rightarrow$ Output Prefix) for $I V_{i}$. In the on-line phase of the attack, the attacker waits until $I V_{i}$ is used for some $i$ and applies the TMTO attack with the tables prepared for that $I V_{i}$.

\footnotetext{
${ }^{2}$ At this stage we consider only attacks mounted on a single key. The scenario in which the attack is mounted on several secret keys simultaneously and the attacker is satisfied with retrieving only one out of the several secret keys is discussed in Section 3.1.

${ }^{3}$ We note that possibly this idea is implicitly mentioned in Section 8 of [7]. In addition, our attack can be considered as a variant of the BSW sampling originally applied in $[5,6]$ to Scenario A attacks on stream ciphers. However, this is the first paper which analyses this approach to Scenario B attacks.
} 
Using the notations introduced in Section 2, the time complexity of the attack is $t^{2}$ and the memory requirement is $m t V / D$. The tables cover most of the key space, and thus $m t^{2}=K$. Hence, the resulting tradeoff curve is $T M^{2}=$ $t^{4} m^{2} V^{2} / D^{2}=K^{2} V^{2} / D^{2}=(K V)^{2} / D^{2}$, or equivalently $T M^{2} D^{2}=(K V)^{2}$.

Using the previous approach, i.e., trying to invert the function $(($ Key,IV $) \rightarrow$ Output Prefix), results in the same tradeoff curve $T M^{2} D^{2}=(K V)^{2}$. However, it appears that the new approach has several important advantages over the former one:

1. Attacking Ciphers with long IVs: Since in the new approach the underlying TMTO attack does not use multiple data points, the tradeoff curve $N^{2}=T M^{2} D^{2}$ can be obtained without restrictions on the parameters, i.e., even for $T<D^{2}$. Hence, assuming $T=M=D$, the complexity of the attack is less than that of exhaustive key search as long as $N^{2}<K^{5}$, or equivalently, $V^{2}<K^{3}$. Therefore, if the key length is $n$ bits, the cipher offers $n$-bit security w.r.t. TMTO attacks only if the IV length is at least $1.5 n$ bits. In the current approach, the parameters of the attack must satisfy the inequality $M T \geq N$ (see [4,6]), and hence if the IV length is at least $n$ bits, either the time or the memory complexity of the attack is not less than $2^{n}$, regardless of the amount of available data. Moreover, since in the former approach the optimal curve can be obtained only for $T \geq D^{2}$ (see $[4,6]$ ), the new approach leads to a better tradeoff curve whenever the amount of available data is greater than $2^{n / 2}$, for an $n$-bit secret key. This advantage is particularly significant in modern stream ciphers, where the IV size is large, where the new approach is able to use more available data then the previous one.

2. Optimal tradeoff for multiple data points in the Rainbow scheme: The new approach allows to use Rainbow tables in the attack instead of the standard Hellman's tables, without losing the advantage of the Rainbow's tradeoff curve. This is possible since the underlying TMTO attack does not use multiple data points at all (which prevents using the full power of the rainbow tables $[2,4])$. Therefore, the new approach allows to use the Rainbow tables also in attacks on stream ciphers with IVs, gaining an improved time complexity and reduction in the rate of false alarms [15]. ${ }^{4}$

3. Reduction in the memory requirements: The new approach reduces the memory requirements of the attack. Since the function we try to invert is (Key $\rightarrow$ Output Prefix), the number of memory bits needed for each chain in the table is $2\lceil\log K\rceil$. When inverting ((Key,IV) $\rightarrow$ Output Prefix) each chain requires $2\lceil\log K+\log V\rceil$ bits. If the IV length is equal to the key length (as recommended in [8]), this difference reduces the memory requirements of the attack by factor 2 .

\footnotetext{
${ }^{4}$ As noted in [2], the advantage of the Rainbow scheme over the standard Hellman's scheme can be questioned. The comparison between the two schemes is out of the scope of this paper. Our claim is that using our technique, both schemes can be used also in attacks on stream ciphers.
} 
4. Reduction in the duration of active eavesdropping: In some cases the new approach allows to reduce the duration of active eavesdropping performed in the attack. Assume that only the first IV is randomly chosen, and then the IV is incremented sequentially (as recommended in [8]). In this case, the attacker can examine the first used IV and find out when one of the chosen IVs will be used. Then, the attacker has to eavesdrop only when the required IV is used. In the standard approach, such an attack requires eavesdropping to all the data.

5. Exploiting uneven distribution of the IVs: If the IVs are not chosen properly, i.e., the distribution of the IVs is not uniform, the new approach allows to exploit this weakness easily. The attacker prepares the tables for the IVs that are most frequently used, and thus reduces the memory and data complexities of the attack. ${ }^{5}$ For example, if the first used IV is known to the attacker in advance (e.g., fixed to zero), the attacker prepares the tables only for this IV, and thus reduces the effect of the IV on the cipher's security. It seems that the standard approach also can exploit improper usage of IVs, but the algorithm becomes more complicated.

On the other hand, the new approach has one drawback: The success probability of the attack might be lower than the success probability in the former approach. In our new approach, the attack succeeds if the following two conditions are satisfied:

1. Amongst the $D$ encryptions available to the attacker, there is an encryption using one of the $V / D$ chosen IVs.

2. The secret key used with the chosen IV is covered by the tables prepared for this IV.

Assuming the attacker chooses the parameters such that $N=T M^{2}$ (or $N=T M^{2} D^{2}$ ), the probability that the obtained sample is covered by one of the tables is about $63 \%$. If all the IVs are chosen randomly and independently, then the probability that one of the IVs that are covered is encountered in the $D$ samples is $63 \%$, and hence, the total success probability of our approach in this case is $40 \%$. For comparison, the standard approach yields a success rate of $63 \%$ while using $\lceil\log K+\log V\rceil /\lceil\log K\rceil$ times more memory. When the size of the key and the IV is the same, both approaches yield $40 \%$ success rate for the same amount of memory.

However, in the majority of protocols where stream ciphers are used with IVs, the first IV is chosen randomly (or even in a deterministic manner), and the IV is incremented in a deterministic manner between the different packets. In this case, by choosing the IVs appropriately, the attacker can resolve the second issue. Thus, in most of the applications, it is expected that the success rate of the new approach is equal to that of the former approach while the memory requirements are reduced.

\footnotetext{
${ }^{5}$ A similar approach was used in [5], under the name biased birthday attack.
} 


\subsection{Attacking Several Secret Keys Simultaneously}

If the attacker mounts the attack on several secret keys simultaneously and is satisfied with retrieving only one of them, the TMTO attack benefits from the ability to use multiple data points. In the standard approach, where the inverted function is ( $(\mathrm{Key}, \mathrm{IV}) \rightarrow$ Output prefix $)$, encryptions under different keys can be naturally used as multiple data points in the attack. In the new approach, the attack strategy depends on the amount of available data.

Assume that the data available to the attacker consists of encryptions under $D_{K}$ different keys, where each key is encrypted under $D_{V}$ IVs. In this case, the tradeoff curve obtained by the standard approach is $T M^{2}\left(D_{K} D_{V}\right)^{2}=K^{2} V^{2}$.

In the new approach, if $D_{K} D_{V} \leq V$, the attacker chooses $V / D_{K} D_{V}$ IVs and pre-computes the tables for inverting the function (Key $\rightarrow$ Output prefix) for each of the chosen IVs. The data contains an encryption using one of the chosen IVs, and once such instance is encountered, the corresponding key can be retrieved.

If $D_{K} D_{V} \geq V$, the attacker chooses a single IV and prepares the tables for it. However, since it is expected that the data contains $D_{K} D_{V} / V$ encryptions using the chosen IV, the constructed tables have to cover only $K /\left(D_{K} D_{V} / V\right)$ of the possible keys, and hence the memory complexity of the attack is reduced. Note that since in this case the underlying attack is a TMDTO attack, the optimal tradeoff curve can be obtained only if $T \geq\left(D_{K} D_{V} / V\right)^{2}$.

In both cases, the obtained tradeoff curve is $T M^{2}\left(D_{K} D_{V}\right)^{2}=K^{2} V^{2}$, as in the standard algorithm. In the case $D_{K} D_{V} \leq V$, all the advantages of the new approach are preserved. In the case $D_{K} D_{V} \geq V$, the first two advantages are weakened, while the other three are preserved. ${ }^{6}$ Therefore, the new approach is better than the former one also in the multiple key case.

\section{Summary and Conclusions}

In this paper we presented a new approach to Time-Memory-Data tradeoff attacks on stream ciphers with initial value. Instead of treating the IV as part of the secret key material and trying to invert the function ((Key,IV) $\rightarrow$ Output prefix), we propose to fix several IVs in advance and attempt to invert the function (Key $\rightarrow$ Output prefix). We showed that while the resulting tradeoff curve in the new approach is the same as the curve obtained by the former approach, the new approach has several advantages over the former one, such as less restrictions on the parameters of the tradeoff.

We conclude that the new approach allows to exploit (to some extent) the fact that the IV is known to the attacker. It is tempting to find further ways to exploit this knowledge, and thus to reduce the influence of the IV on the cipher's security. We expect these results to affect the way IVs are used in various protocols, to counter our findings.

\footnotetext{
${ }^{6}$ On the other hand, for $D_{K} D_{V} \gg V$, the success probability of the new approach is equal to that of the standard approach, since the chosen IV appears in the data with overwhelming probability.
} 


\section{References}

1. Steve H. Babbage, Improved "exhaustive search" attacks on stream ciphers, IEE European Convention on Security and Detection, IEE Conference publication 408, pp. 161-165, IEE, 1995.

2. Elad Barkan, Eli Biham, Adi Shamir, Rigorous Bounds on Cryptanalytic Time/Memory Tradeoffs, Advances in Cryptology, proceedings of Crypto 2006, Lecture Notes in Computer Science 4117, pp. 1-21, Springer-Verlag, 2006.

3. Elad Barkan, Cryptanalysis of Ciphers and Protocols, Ph.D. Thesis, Technion, 2006.

4. Alex Biryukov, Sourav Mukhopadhyay, Palash Sarkar, Improved Time-Memory Tradeoffs with Multiple Data, proceedings of Selected Areas in Cryptography 2005, Lecture Notes in Computer Science 3897, pp. 245-260, Springer-Verlag, 2006.

5. Alex Biryukov, Adi Shamir, David Wagner, Real Time Cryptanalysis of A5/1 on a $P C$, proceedings of FSE 2000, Lecture Notes in Computer Science 1978, pp. 1-18, Springer-Verlag, 2001.

6. Alex Biryukov, Adi Shamir, Cryptanalytic Time/Memory/Data Tradeoffs for Stream Ciphers, Advances in Cryptology, proceedings of ASIACRYPT 2000, Lecture Notes in Computer Science 1976, pp. 1-13, Springer-Verlag, 2000.

7. Johan Borst, Bart Preneel, Joos Vandewalle, On the Time-Memory Tradeoff Between Exhaustive Key Search and Table Precomputation, proceedings of the 19th Symposium on Information Theory in the Benelux, Veldhoven (NL), pp. 111-118, 1998.

8. Christophe De Canniere, Joseph Lano, and Bart Preneel, Comments on the Rediscovery of Time Memory Data Tradeoffs, eSTREAM, ECRYPT Stream Cipher Project, Report 2005/040.

9. ECRYPT, Call for Stream Cipher Primitives, version 1.3, 12.4.2005. Available online at http://www.ecrypt.eu.org/stream/call/.

10. Amos Fiat, Moni Naor, Rigorous Time/Space Trade-offs for Inverting Functions, SIAM Journal of Computing, vol. 29, No. 3, pp. 790-803, 1999.

11. Jovan Dj. Golic, Cryptanalysis of Alleged A5 Stream Cipher, Advances in Cryptology, proceedings of EUROCRYPT '97, Lecture Notes in Computer Science 1233, pp. 239-255, Springer-Verlag, 1997.

12. Martin E. Hellman, A Cryptanalytic Time-Memory Tradeoff, IEEE Transactions on Information Theory, Vol. 26, No. 4, pp. 401-406, 1980.

13. Jin Hong, Palash Sarkar, Rediscovery of Time Memory Tradeoffs, IACR Eprint Report 2005/090, 2005. Available on-line at http://eprint.iacr.org/2005/090.

14. Jin Hong, Palash Sarkar, New Applications of Time Memory Data Tradeoffs, Advances in Cryptology, proceedings of Asiacrypt 2005, Lecture Notes in Computer Science 3788, pp. 353-372, Springer-Verlag, 2005.

15. Philippe Oechslin, Making a Faster Cryptanalytic Time-Memory Trade-Off, Advances in Cryptology, proceedings of CRYPTO 2003, Lecture Notes in Computer Science 2729, pp. 617-630, Springer-Verlag, 2003.

16. Markku-Juhani Olavi Saarinen, A Time-Memory Tradeoff Attack Against LILI128, proceedings of FSE 2002, Lecture Notes in Computer Science 2365, pp. 231236, Springer-Verlag, 2002.

17. Michael J. Wiener, The Full Cost of Cryptanalytic Attacks, Journal of Cryptology, Vol. 17, No. 2, pp. 105-124, 2004. 